\title{
CAPILLAROSCOPY - A VALUABLE TOOL FOR THE ASSESSMENT OF VASCULAR IMPAIRMENT IN SYSTEMIC SCLEROSIS
}

\author{
Cristina Criveanu ${ }^{1,2}$, Paulina Lucia Ciurea ${ }^{1,2}$, Anca Emanuela Musetescu ${ }^{1,2}$, Andreea Barbulescu ${ }^{3}$, \\ Sineta Firulescu ${ }^{2}$, M. Florea ${ }^{2}$, A.F. Vreju ${ }^{1,2}$ \\ ${ }^{\prime}$ Department of Rheumatology, Emergency County Hospital, Craiova \\ ${ }^{2}$ Department of Rheumatology, University of Medicine and Pharmac, Craiova \\ ${ }^{3}$ Department of Pharmacology, University of Medicine and Pharmacy, Craiova
}

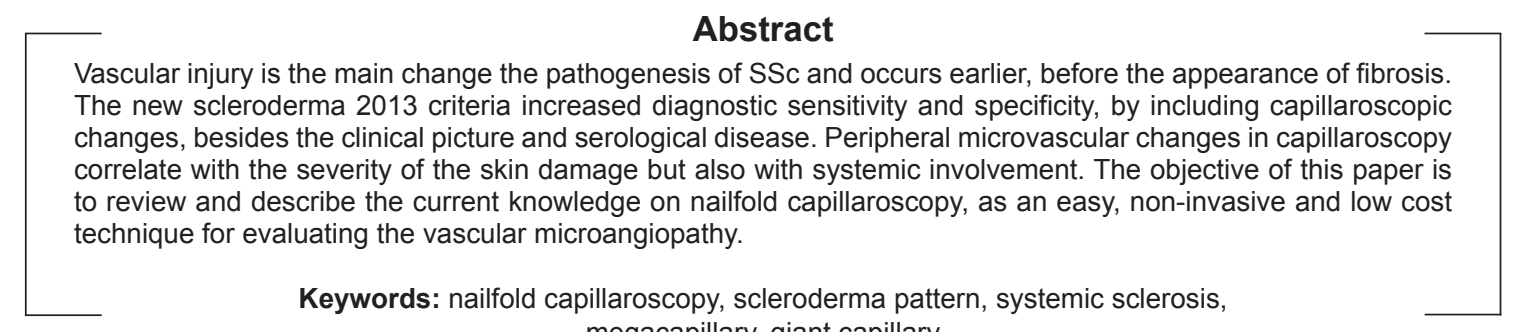
megacapillary, giant capillary

\section{INTRODUCTION}

Systemic sclerosis (SSc) is a chronic, multisystem disabling connective tissue disorder characterized by endothelial and fibroblast dysfunction and accumulation of extracellular matrix on the blood vessels, skin and viscera.

Vascular injury is "primum movens" in the pathogenesis of SSc and occurs earlier, before the appearance the fibrosis and its histopathological evidence can be identified both in the affected and non-affected skin (1). The first change is represented by vascular intima thickening, in small and medium arteries, due to increased proliferation and myointimal cell migration and accumulation of collagen (2).

Vascular basal lamina is thickened, alteration of fibrinolysis occurs, levels of von Willebrand factor increase. Endothelial cell injury is a consequence of continuous platelet aggregation with platelet-derived growth factor (PDGF) and endothelin-1 (ET-1) release, followed by endothelial cell apoptosis $(3,4)$. Other factors with role in pathogenesis include autoantibodies against endothelial cells, vascular tropic viruses or inflammatory cytokines. Lesions vasculi- tis-like with deposition of immune complexes are uncommon.

In advanced stages of the disease, extensive fibrin deposition and perivascular fibrosis cause progressive occlusion of the arterial lumen with marked reduction in the number of small vessels and chronic hypoxia in damaged tissues (5).

Vascular injury leads to activation and endothelial cell dysfunction, with increased expression of cell adhesion molecule 1 and endothelial leukocyte adhesion molecule 1, modifying vasoactive mediators' secretion and platelet activation and fibrinolysis pathway $(6,7)$. Thus, activated platelets release thromboxane A2, PDGF and TGF- $\beta$, which initiates vasoconstriction and contributes to the activation of fibroblasts and myofibroblasts differentiation. It also increases the number of pericytes expressing surface markers Thy 1 (CD90) and PDGF receptors $(8,9)$.

Endothelial cell response to vasodilators factors (prostacyclin, nitric oxide) are ineffective, the gain of vascular injury being aggravated by alteration of vasodilation/vasoconstriction balance and episodes of ischemia/reperfusion. The consequences are: in-

\footnotetext{
Correspondence address:

Ananu Florentin Vreju, Lecturer, MD, PhD, MSc, Department of Rheumatology, University of Medicine and Pharmacy Craiova,

2-4 Petru Rares Street, Craiova

E-mail: florin_vreju@yahoo.com
} 
creased capillary permeability and leukocytes migration, platelet and fibrinolysis pathway activation with the occurrence of thrombosis.

The activated endothelial cells release ET-1, the most powerful vasoconstrictor that promotes leukocyte and vascular smooth muscle cells adhesion, followed by activation of fibroblasts.

Hypertrophy of the intima and the medium layer of the wall of small vessels and adventitia fibrosis causes progressive narrowing of the lumen, which, associated with endothelial cell apoptosis, leads to reduction of number of blood vessels (10), tissue chronic hypoxia and increased expression of VEGF and its receptors in damaged tissues (11-13). It has been showed that serum concentrations of endostatin inhibitor of angiogenesis is elevated and a degradation product of collagen type XVIII is often found in SSc patients (14).

Microvascular injury is characterized by a decrease in capillary number and structure change, leading to a decrease in blood flow and tissue ischemia, the end state being the avascular areas and formation of giant capillaries, in an attempt to support tissue nutrition. All these changes can be assessed by morphological vascular capillaroscopy.

Nailfold capillaroscopy is a non-invasive method for the assessment of capillaries number and morphology, by examining the nail fold. Capillaroscopy is an important imaging tool used for microvascular study with an important benefit in early assessment of systemic sclerosis and in the same time.

\section{Brief history of capillaroscopy}

The first visualization of capillaries was made in 1661 by Marcello Malpighi. Italian physician Giovanni Rasori (1766-1837), described a relationship between conjunctive tissue inflammation and the presence of "knots of capillary loops", visible through a magnifying glass (15). After this description, in the early twentieth century, Sir Jonathan Hutchinson differentiated primary from secondary Raynaud's phenomenon (16).

In 1912, Lombard examined nail fold capillaries using a microscope by applying a drop of oil. In 1918, Weiss has perfected the capillaroscopy technique and took images using a camera. In 1922, Otfried Müller published the first color atlas of changes in the capillaries viewed in the nail fold. In 1925, Brown and O'Leary demonstrated microvascular changes in Raynaud's phenomenon in scleroderma using capillaroscopic analysis. After these observations, capillaroscopy has been neglected for several decades, until the second half of the twentieth century, when Hildegard Maricq, considered a pioneer of modern capillaroscopy, along with LeRoy Carwile described capillaroscopic patterns in SSc. Fagrell studied capillaries in foot of patients with chronic venous insufficiency and in 1979 - Bollinger (Zürich) introduced fluorescent tracers technique using a solution of $20 \% \mathrm{Na}$ - fluorescein in bolus injections, in the antecubital veins, thus achieving transcapillary and interstitial diffusion.

In 2001 capillaroscopy abnormalities were included in defining systemic sclerosis subsets by LeRoy and it was suggested their importance in its early diagnosis (17).

Nailfold capillary microscopy can be made with several kinds of optical instruments, such as the ophthalmoscope, conventional optical microscope, stereo microscope, videocapillaroscope.

\section{Normal and pathological capillaroscopy items}

In capillaroscopic assessment two parameters must be followed: morphological (static) and functional (dynamic) changes.

For the morphological criteria there have been considered the following factors: visibility, capillary loops morphology, size (diameter) of capillary loops, loops length and their distribution, capillary density and microbleeds. Functional criteria evaluate the type of flow, as continuous or normal, granular aspect, microaggregates, flashing or "goals", with or without interruptions.

Capillaroscopy also offers data about quality of extracellular matrix (transparent, color, presence of edema, hemosiderin deposits, bleeding areas).

Visibility depends on cutaneous transparency and skin thickness varying with sex, age and profession. The decrease of skin transparency diminishes the visibility of the capillary network and this is due, in early stages of the disease, to interstitial oedema, and in more advanced stages to extracellular matrix excess. In $30 \%$ of the healthy subjects, venous plexus is more visible in the $4^{\text {th }}$ and $5^{\text {th }}$ finger. In certain conditions it may be irregular, enlarged and twisted.

The morphology of normal capillary loops shows a "U" or hairpin aspect, parallel to the skin surface of cuticle. Morphology loops can vary depending on the subject's age, physiological abnormalities being often present: tortuosity, with one or more crossings, 
in the shape of "8", "corkscrew" Branched capillaries ("clover", "cactus", "fern") and "bush-like" ones, or ectasia loops, regular or irregular, diffuse or circumscribed, apical or lateral are more frequently pathological.

Very closely correlated with these changes is loops size. The normal diameter loops varies between $5-15 \mu \mathrm{m}$, the arterial branch is usually thinner than the venous one. The apical side has a diameter between $8-14 \mu \mathrm{m}$. If the arterial limb is larger than $15 \mu \mathrm{m}$ or the venous one is wider than $20 \mu \mathrm{m}$, we can consider that an abnormal, dilated capillary. Homogeneously dilated loops can reach a diameter of $>50$ micrometers - giant capillaries, or can reach a diameter of 100-200 $\mu \mathrm{m}$, and are called megacapillaries. Microbleeding occurs very often near megacapillaries and along with other changes define scleroderma patterns.

Capillary length is a parameter harder objectified, depending on skin transparency, generally ranging between 200-500 $\mu \mathrm{m}$.

Microhaemorrhages can occur as a consequence of the disruption of the vessel wall, being viewed most frequently in the last row of the capillaries. Microhaemorrhage may be recent, not very well defined, appearing as a red spot near the top bleeding capillary loop, or near the branches (bleeding side), or older, deeper, darker and more distant of loop origin, that can turn brown in time into a hemosiderin deposit along the edge of the skin. Microhaemorrhages are a pathognomonic scleroderma element, especially along with megacapillaries.

Capillary density is determined by the number of capillary loops per linear millimetre. Normally, the density varies between 10 to 15 capillaries $/ \mathrm{mm}$, each dermal papilla containing a capillary loop. The decrease of capillary density network is frequently seen in SSc spectrum diseases, as a consequence of microvascular disease. The disappearance of a loop (drop-out) may expand, forming avascular areas with absence of capillaries on area $>500 \mu \mathrm{m}$ and "desert-like" aspect. It is important to differentiate those avascular areas from those with just poor visibility because of diffuse cutaneous oedema. Neovascularization, with formation of giant capillaries, compensates for the decreasing number of loops. Losing the capillary loops is associated with active forms of the disease and with a poor prognosis (18).

Finally, capillaroscopy can differentiate by the dynamic parameter, a flow feature, between the regular and normal flow through the loops and the gran- ular one, with the presence of microaggregates inside the loop, with intermittent, discontinuous aspect and low flow. A typical change scleroderma capillary flow is the so-called phenomenon "sludge", "plasma penetration".

\section{Capillaroscopy pattern in scleroderma}

Raynaud's phenomenon is the most common claim that requires microcirculation analysis, capillaroscopy being able to help distinguish between primary and secondary disease. Changes in SSc peripheral microvasculature are characterized by the presence of structural capillaries changes (megacapillaries and haemorrhage), with a progressive decrease in the number of capillary loops.

The changes were first described by HR Maricq as a combination of very dilated capillaries, avascular areas and bleeding, with disorganization of the normal architecture of the nailfold capillaries (19). Subsequently, he defined two types of scleroderma patterns: active - which associated megacapillaries with decreased density loops and it correlated with diffuse cutaneous scleroderma and slow pattern, with giant capillaries, but keeping the capillary loops, associated with limited cutaneous scleroderma and better prognosis (20).

Alterations in microvascular nailfold of SSc were described and classified by Maurizio Cutolo, based on capillaroscopic vessels appearance and depending on the progression of the disease, in three patterns: early, active and late. This classification reflects the evolution of the SSc microangiopathy and the recognition of three capillaroscopic models (Fig. 1) could help on staging and assessment of disease activity:

1. "Early pattern" is characterized by the appearance of several dilated capillaries/megacapillaries, some bleeding, without loss of capillary loops.

2. "Active pattern" shows frequent bleeding megacapillaries, moderate loss of capillaries, moderate disorganization of the capillary architecture, rare/absent branching capillaries.

3. "Late pattern" defined by severe loss of capillaries, neovascularization by megacapillaries, no hemorrhages and massive avascular areas.

\section{Capillaroscopy in modern era}

The new 2013 ACR and EULAR criteria (21) for the classification of scleroderma, increased diagnos- 


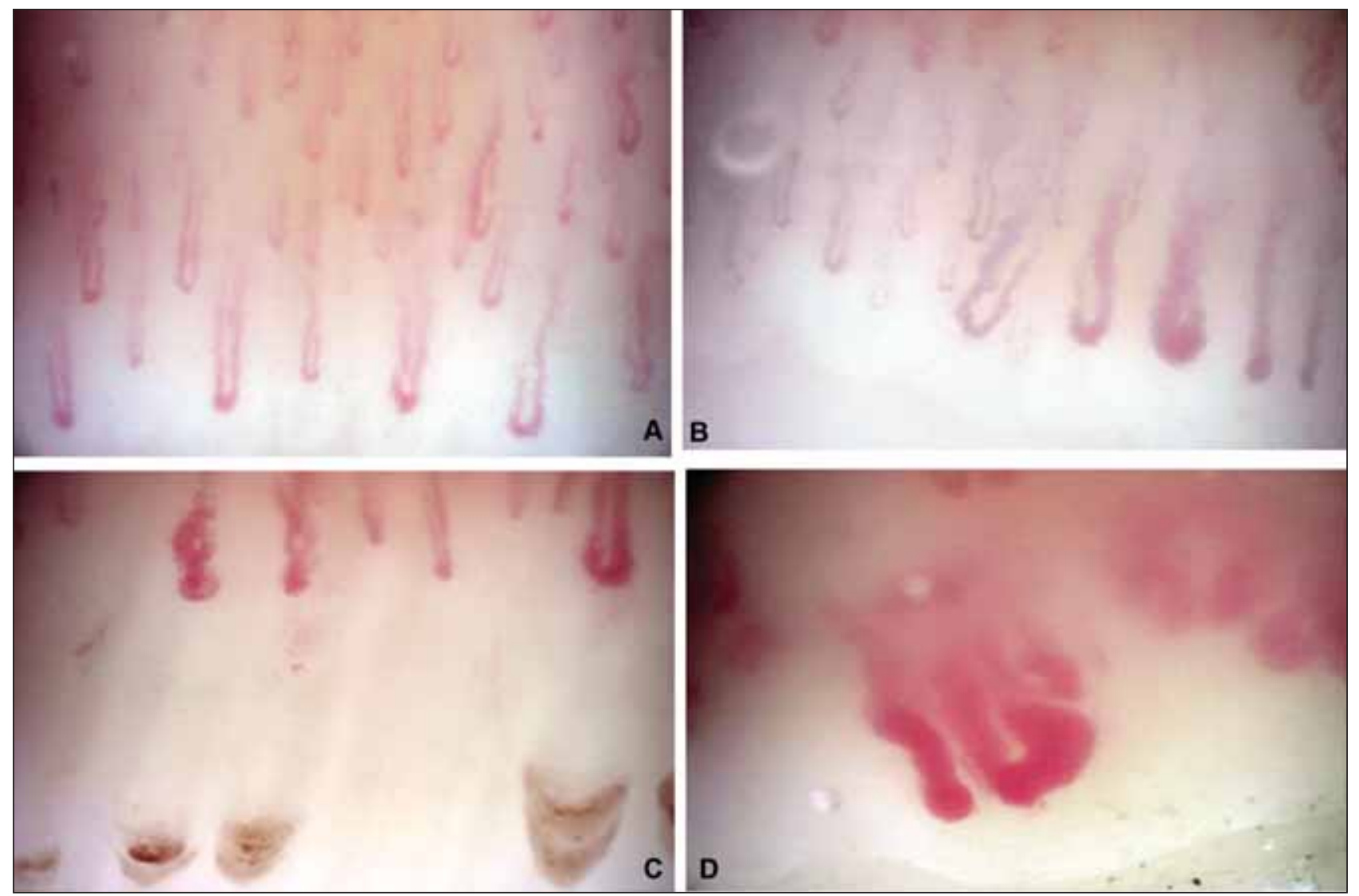

FIGURE 1. A. Normal pattern; B. Early pattern - several dilated capillaries, no loss of capillary loops; C. Active pattern bleeding capillaries, moderate disorganization of the capillary architecture; $D$. Late pattern - loss of capillaries, neovascularization by megacapillaries, no hemorrhages

tic sensitivity (91\%) and specificity (92\%), by including capillaroscopic changes, besides the clinical picture and serological disease and thus, demonstrating once again the importance of this method in evaluating and early diagnosis of the disease. However, in clinical practice, the new criteria are mostly used in clinical trials and are not considered diagnosis criteria; patients with Raynaud's phenomenon, autoantibodies or capillaroscopic changes, can be diagnosed with SSc, without meeting the new ACR/ EULAR criteria (22).

Despite some controversial results, an important number of studies have demonstrated that peripheral microvascular changes in capillaroscopy correlate with the severity of the skin damage but also with systemic involvement; thus since 1976, Maricq et al. revealed a correlation between the number of involved organs and capillaroscopic abnormalities (23).

Microvascular changes such as those assessed by nailfold capillaroscopy are seen in all involved organs (lung, heart, kidneys and gastrointestinal tract) (24).

The most severe complication and the leading cause of mortality in patients with SSc, pulmonary arterial hypertension (PAH), is a disease of the pulmonary arterioles, characterized by proliferation and vascular remodeling, followed by progressively increasing pulmonary vascular resistance (PVR), with right ventricular failure (passing successively through the stages of hypertrophy and dilatation) and finally, possibly exitus. An Asian study, from 1998, demonstrated a correlation between the reduction in capillary density, presence of dilated capillaries, branched and structural disorganization in SSc patients and $\mathrm{PAH}$, demonstrating the importance of capillaroscopy in the management of the disease (26).

Digital ulcers are a frequent complication of SSc, with an important impact on the quality of life. The importance of capillaroscopic findings in predicting digital ulcers, was demonstrated by Sebastiani et al. by calculating the capillaroscopic skin ulceration risk index (CSURI) DxM/N $\mathrm{N}^{2}$ (D- diameter of the largest giant loop, M- number of giant loops, Nnumber of all loops) (27).

Capillaroscopic patterns were associated with different subsets of SSc. Severity of vascular, skin, heart or lungs involvement, increases progressively 
from being "early" to "active" and then to "late", patients with the latter pattern, having an increased risk of severe active disease $(28,29)$ and cardiac and pulmonary involvement (26). Also, renal involvement was correlated with a capillaroscopic pattern which is characterized by avascular areas and architectural disorganization (30-32).

\section{CONCLUSIONS}

Systemic sclerosis is an autoimmune disorder characterized by early impairment of microvascular

\section{REFERENCES}

1. Freemont A.J., Hoyland J., Fielding P., et al. Studies of the microvascular endothelium in uninvolved skin of patients with systemic sclerosis: Direct evidence for a generalized microangiopathy. Br J Dermatol 1992; 126:561-568

2. Prescott R.J., Freemont A.J., Jones C.J., et al. Sequential dermal microvascular and perivascular changes in the development of scleroderma. J Pathol 1992; 166:255-263.

3. Kahaleh M.B. Endothelin, an endothelial-dependent vasoconstrictor in scleroderma: Enhanced production and profibrotic action. Arthritis Rheum 1991; 34:978-983.

4. Varga J., Heiman-Patterson T.D., Emery D.L., et al. Clinical spectrum of the systemic manifestations of the eosinophilia-myalgia syndrome. Semin Arthritis Rheum 1990; 19:313-328.

5. Yousem S.A. The pulmonary pathologic manifestations of the CREST syndrome. Hum Pathol 1990; 21:467-474

6. Cerinic M.M., Valentini G., Sorano G.G., et al. Blood coagulation, fibrinolysis, and markers of endothelial dysfunction in systemic sclerosis. Semin Arthritis Rheum 2003; 32:285-295.

7. Hummers L.K. Microvascular damage in systemic sclerosis: Detection and monitoring with biomarkers. Curr Rheumatol Rep 2006; 8:131-137.

8. Helmbold P., Nayak R.C., Marsch W.C., et al. Isolation and in vitro characterization of human dermal microvascular pericytes. Microvasc Res 2001; 61:160-165.

9. Rajkumar V.S., Sundberg C., Abraham D.J., et al. Activation of microvascular pericytes in autoimmune Raynaud's phenomenon and systemic sclerosis. Arthritis Rheum 1999; 42:930-941.

10. Fleming J.N., Nash R.A., McLeod D.O., et al. Capillary regeneration in scleroderma: Stem cell therapy reverses phenotype?. PLOS ONE 2008; 16(31):e1452.

11. Davies C.A., Jeziorska M., Freemont A.J., et al. The differential expression of VEGF, VEGFR-2, and GLUT-1 proteins in disease subtypes of systemic sclerosis. Hum Pathol 2006; 37:190-197.

12. Distler O., Distler J.H., Scheid A., et al. Uncontrolled expression of vascular endothelial growth factor and its receptors leads to insufficient skin angiogenesis in patients with systemic sclerosis. Circ Res 2004; 95:109-116.

13. Distler O., Del Rosso A., Giacomelli R., et al. Angiogenic and angiostatic factors in systemic sclerosis: Increased levels of vascular endothelial growth factor are a feature of the earliest disease stages and are associated with the absence of fingertip ulcers. Arthritis Res 2002; 4:R11

14. Hebbar M., Peyrat J.P., Hornez L., et al. Increased concentrations of the circulating angiogenesis inhibitor endostatin in patients with systemic sclerosis. Arthritis Rheum 2000; 43:889-893.

15. Maurizio Cutolo, carmen Pizzorni, Alberto Sulli, Capillaroscopy, Best Practice and research Clinical Rheumatology, vol 19, no 3, pp 437-452, 2005

16. Maurizio Cutolo, Carmen Pizzorni, Alberto Sulli, Capillaroscopy, Best Practice and research Clinical Rheumatology, 2008, 22: 1093-1108

17. LeRoy E.C., Black C., Fleischmajer R., et al. Scleroderma (systemic sclerosis): classification, subsets and pathogenesis. J. Rheumatol 1988, 15:202-205). system. The best method to evaluate the vascular microangiopathy is the nailfold videocapillaroscopy an easy, safe, non-invasive and low cost technique for diagnosing pathologies of the peripheral microcirculation and it is a valuable tool in the rheumatologists' daily practice.
18. Anderson M.E., Allen P.D., Moore T., Hillier V., Taylor C.J., Herrick A.L. Computerised nailfold video capillaroscopy - a new tool for the assessment of Raynaud phenomenon. J Rheumatol 2005; 32:841-8

19. Maricq H.R., Weinberger A.B., LeRoy E.C. Early detection of scleroderma-spectrum disorders by in vivo capillary microscopy: a prospective study of patients with Raynaud phenomenon. J Rheumatol 1982, 9: 289-291

20. Maricq H.R. Widefield capillary microscopy. Technique and rating scale for abnormalities seen in scleroderma and related disorders. Arthritis Rheum 1981, 24: 1159-1165

21. Van der Hogen F. et al. Classification Criteria for Systemic Sclerosis: An American College of Rheumatology/European League Against Rheumatism Collaborative Initiative† Arthr Rheum 2013, 65, 2737-2747, 2013

22. Jordan S., Maurer B. et al. Performance of the new ACR/EULAR classification criteria for systemic sclerosis in clinical practice. Rheum 2015; 54:1454-1458

23. Maricq H.R., Spencer-Green G., LeRoy E.C. Skin capillary abnormalities as indicators of organ involvement in scleroderma. Raynaud's syndrome and dermatomyositis. Am J Med. 1976; 61

24. Grassi W., Core P., Carlino G. et al. Labial capillary microscopy in systemic sclerosis. Ann Rheum Dis. 1993; 52(8), 564-569

25. Ong Y.Y., Nikoloutsopoulos Ti., Bond C.P., Smith M.D., Ahern M.J., Roberts-Thomson P.J. Decreased nailfold capillary density in limited scleroderma with pulmonary hypertension. Asian Pac $J$ Allergy Immunol 1998; 16(2-3):81-6

26. Hofstee H.M., Noordegraaf A.V., Voskuyl A.E., Dijkmans B.A., Postmus P.E., Smulders Y.M. et al. Nailfold capillary density is associated with the presence and severity of pulmonary arterial hypertension in systemic sclerosis. Ann Rheum Dis 2009; 68:191-5

27. Sebastini M. et al. Capillaroscopic skin ulcer risk index: a new prognostic toolfor digital ulcer developement in systemic sclerosi patients. Arthrits Rheum 2009; 61:688-694)

28. Sambataro D., Sambataro G. et al. Naildfold videocapillaroscopy micro-haemorrhage and giant capillary counting as an accurate approach for a steady state definition of disease activity in systemic sclerosis. Arthritis Research\&Therapy 2014, 16:462

29. Hoanca C., Vreju F. et al. Corelatii intre ulceratiile digitale si aspectele clinico-biologice la pacientii cu sclerodermie. Medicina Interna 2013, vol X, nr 4 ISSN 1220-5818 pag 17-23

30. Rosato E., Gigante E., Barbano E. et al. Intrarenal hemodynamic parameters correlate with glomerular filtration rate and digital microvascular damage in patients with systemic sclerosis. Semin Arthritis Rheum 1996; 39(6):1030-4

31. Hoanca C. et al. Correlations between scleroderma renal crisis and videocapillaroscopic patterns in patients with systemic sclerosis. Current Health Sciences Journal, Vol 38, No 4, October-December 2012

32. Caraba A., Crisan V., munteanu A. et al. Assessment of inrarenal vascularization in diffuse systemic sclerosis. Roumanian Journal of Rheumatology, vol XXIV, no 3, 2015 